\title{
TISSUE DISTRIBUTION OF B-LYMPHOCYTE SUBSETS (IGA, IGG AND IGM) IN THE MUCOSA AND LYMPHOID TISSUES OF BROILERS IMMUNIZED WITH GUMBORO VACCINE
}

\author{
M. T. Hussan ${ }^{2}$, M. Z. I. Khan ${ }^{1}$ and M. R. Jahan ${ }^{1}$ \\ ${ }^{1}$ Department of Anatomy and Histology, Faculty of Veterinary Science, Bangladesh Agricultural University, \\ Mymensingh-2202, Bangladesh. \\ ${ }^{2}$ Department of Anatomy and Histology, Faculty of Animal Science and Veterinary Medicine, Patuakhali \\ Science and Technology University, Babugonj, Barisal-8210, Bangladesh.
}

\begin{abstract}
The present research was designed to study the histological and immunohistochemical changes of lymphoid (bursa of Fabricius and cecal tonsil) and mucosal (ileum) tissues of broiler chickens after immunization with Gumboro vaccine. Two groups $(n=24)$ of Cobb-500 broiler chickens were reared in the same environment. A mild vaccine (BUR $\left.{ }^{\circledR} 706\right)$ followed by an intermediate plus (Nobilis ${ }^{\circledR}$ Gumboro $228 \mathrm{E}$ ) vaccine was administered for immunization of chickens and samples (bursa of Fabricius, cecal tonsil and ileum) were collected at 7 days interval for up to 32 days of age. In the bursa of Fabricius, the population of lymphocytes and the size of the follicles were found to increase in vaccinated chickens than the control chickens. The Igs positive cells (IgA, IgG and IgM) were distributed principally beneath the capsule, around the follicles and in the cortex and medulla of bursa of Fabricius. The frequencies of IgG- and IgM- positive cells were intense than the IgApositive cells in the bursa of Fabricius vaccinated chickens. IgM-positive cells became peak at day 25 and started to decline at day 32 in the bursa of Fabricius of vaccinated groups of chickens. In the cecal tonsils and ileum, the Igs positive cells (IgA, IgG and IgM) were distributed around the intestinal gland and within the lymphatic nodules of the lamina propria, in the core of the villi, and within the epithelium in both the control and vaccinated chickens. Their frequency per $0.1 \mathrm{~mm}^{2}$ area of the lamina propria and in the epithelium was increased abruptly in the vaccinated group than the control broiler chickens. Like bursa, in the cecal tonsil and ileum of vaccinated chickens, the frequencies of IgG- and IgM-positive cells were also abundant than IgA-positive cells. The present study suggested that the Igs positive cells were greatly accelerated in the vaccinated chickens than the control chickens and it may be due to immunomodulatory effect of vaccine.
\end{abstract}

Key words: B lymphocyte, Broilers, Lymphoid tissues, Gumboro vaccine.

\section{INTRODUCTION}

Infectious bursal disease virus (IBDV) is one of the important viruses of poultry, affects the immune system and causes disorganization of the histological structure of the system concerned leading to poor production of meat and egg and even mortality rate of chickens increases significantly. Histopathological lesions of IBDV infection found in most of the lymphoid organs such as bursa of Fabricius, spleen, thymus, Harderian gland and caecal tonsils (Lukert and Saif, 1997). Changes in the bursa of Fabricius are characterized by necrosis and depletion of B-cells in the germinal follicles (Verveldset al., 1998), interfollicular edema and hyperemia, lymphocytic depletion (Rautenschleinet al., 2001), heterophilic infiltration in the interfollicular space (Tanimuraet al., 1995) and also in the follicles (Elankumaran, 2002). Now a day, in order to protect Gumboro disease, the broiler farmers use various commercial vaccines. However, their individual effectiveness, especially in generation, mobilizing and distributing the immunocompetent cells (lymphocytes and IgA, IgG and IgMcontaining plasma cells) in the lymphoid and mucosal organs is not clear. Knowledge about the kinetics and Igs class distribution of an antibody response in the tissues of lymphoid and mucosal organs might enlarge our understanding of the induction and effectors phase of Gumboro vaccine and eventually provide new information to develop and evaluate vaccines and to optimize vaccine application. Therefore, the present research has been designed to study the histology of the lymphoid (bursa of Fabricius and cecal tonsils) and mucosal organ (ileum) of broiler chickens, and to study the frequency of the population of lymphocytes and plasma cells containing different classes of immunoglobulins ( $\operatorname{IgA}, \operatorname{IgG}$ and $\operatorname{IgM}$ ) in those tissues following vaccination of chickens with infectious bursal disease virus vaccines.

*Corresponding e-mail address: tufazzal84@gmail.com 


\section{T. Hussan and others}

\section{MATERIALS AND METHODS \\ Chickens}

A total 24 (twenty four) day-old "Cobb-500" broiler chickens of both sexes were purchased from "Kazi Farm Ltd."

First sample was collected from 3 chickens at day 3 (pre-vaccination), second sample was collected from chickens at day 11 (pre-vaccination) and remaining chickens $(n=18)$ were divided equally into vaccinated group and control group. First vaccine was administered at the same day (day 11) to 9 broiler chickens using BUR ${ }^{\mathbb{R}}$ 706 vaccine (Merial, France) through intraocular route, 1 drop in each eye. At day 18, sample (n=3) was collected from both group ( $3^{\text {rd }}$ sample) and second dose of vaccine was administered to remaining 6 chickens of vaccinated group using Nobilis ${ }^{\circledR}$ Gumboro 228E (Intervet, The Netherlands) through intraocular route at day 18. Later on fourth and fifth samples were collected at day $25(n=3)$ and day $32(n=3)$, respectively from both vaccinated and control group. For histological and immunohistochemical staining purpose, the bursa of Fabricius, cecal tonsils and ileum was collected, after sacrificing the chickens through cervical subluxation method.

\section{Preparation of samples for histological studies}

For histological studies all the samples were cut into pieces and then fixed in the "Bouin's fluid" (Gridley, 1960), dehydrated in a series of ascending grades of alcohol, cleared in several changes of xylene, and infiltrated with different grades of melted paraffin in the oven. The tissues were then embedded in paraffin and finally the sections were cut at 6- $\mu \mathrm{m}$ thickness using sliding microtome (MIC 509, Euromex, Japan). The sections were then stained with Hematoxylin and Eosin staining method (Gridley, 1960).

\section{Antibodies}

The antibodies for detecting Igs-containing plasma cells used in this experiment were normal rabbit serum (Biosource, Camarillo, California, USA), goat anti-chicken IgA (Bethyl Lab, USA), goat anti-chicken IgG (Bethyl Lab, USA), goat anti-chicken IgM (Bethyl Lab, USA), and HRP-conjugated rabbit anti-goat IgG (Bethyl Lab, USA).

\section{Immunohistochemical staining and histoplanimetry}

Indirect immunoperoxidasestainingmethod was done for the study of the distributional pattern and frequency of the Ig-containing plasma cells in the lymphoid (bursa of Fabricius and cecal tonsils) and mucosal (ileum) organ of vaccinated and control broilers. The tissues were fixed in ice-cold PLP (Periodate-lysineparaformaldehyde), dehydrated in a series of graded alcohol, cleared in xylene, and embedded in paraffin. Paraffin sections, $6 \mu \mathrm{m}$ thickness, were immunostained by the indirect immunoperoxidase method as described earlier (Khan et al., 1997 and 2007). The immunopositive cells (Igs-positive cells) in the lymphoid tissues and mucosa were counted in 20 fields at a magnification of 40 according to Weibel (1969) and their relative frequency per $0.1 \mathrm{~mm}^{2}$ was calculated using ocular micrometer.

\section{Statistical analysis}

All values were expressed as Mean \pm SE. Statistical analyses were performed by independent sample $t$ test. Significance was established when the probability level was equal to or less than $5 \%$.

\section{RESULTS AND DISCUSSION}

In the present research histological and immunohistochemical studies have been carried out to investigate the changes in generating, mobilizing and distributing the immunocompetent cells (lymphocytes and $\operatorname{IgA}$, IgG and IgM containing plasma cells) in the lymphoid (bursa of fabricius and cecal tonsil) and mucosal organ (ileum) of Cobb-500 chickens of Bangladesh after immunized with Gumboro vaccines. In the present study it was found that the bursa was consisting of long thick mucosal folds (plicae) in all groups of chickens which were projected into the lumen. The lining epithelium of the plicae was pseudostratified columnar epithelium except at the apex where it is simple columnar. Each plicae consisting of numerous follicles and each follicle was composed of peripheral cortex and central medulla in both groups of chickens. It was also observed that the population of bursal lymphocytes and the follicular size were found to increase in vaccinated chickens than the control chickens (Fig. 1a-b). The findings were similar to the result of Honjo and Hirota (1993).

In the bursa of Fabricius, immunohistochemical study revealed that the Igs positive cells (IgA, $\operatorname{IgG}$ and $\operatorname{IgM})$ were found principally beneath the capsule and around the follicles and in the cortex and also medulla and these were more in the vaccinated group (Fig. 2a-b) than the control chickens. 
In the control chickens, IgM positive cells were more than the IgA and IgG positive cells (Fig. 5). The findings were similar to the findings of Honjo and Hirota (1993), they described that most of the bursal lymphocytes were IgM positive; a small population of bursal cells were IgG positive and a few cells were IgA positive. In the vaccinated chickens, Igs positive cells were sharply increased than the control chickens and this might be due to immunomodulation of vaccine. IgM and IgG positive cells were abundant than the IgA positive cells in the bursa of vaccinated group of chickens. IgM positive cells were peak at day 25 and started to decline at day 32 in the chickens of vaccinated group (Fig. 5) and these findings were similar to the finding of Rose (1979). Cecal tonsil is a part of mucosa-associated lymphoid tissue (MALT) and it was composed of four histological layers i.e. tunica mucosa, submucosa, muscularis and serosa. Their lining epithelium was simple columnar epithelium. The bases of the mucosal folds (villi) were thick and the apexes were pointed or rounded in all groups of chickens in the present study. It was observed that the lymphocytes in lamina propria and the intraepithelial lymphocytes in the cecal tonsil were greatly accelerated in the vaccinated group than the control group of chickens. The size and number of lymphatic nodules also increased in the vaccinated group. In the control group lymphocytic population increased that was age related change but not as much high as vaccinated group. In the cecal tonsils, Igs positive cells (IgA, IgG and IgM) were distributed within the lymphatic nodules, lamina propria and in the core of the villi in both the control and vaccinated chickens. Their frequency per $0.1 \mathrm{~mm}^{2}$ area of the lamina propria and core of the villi of cecal tonsils increased abruptly in the vaccinated group than the chickens of control group (Fig. 3a-b). In the control chickens, IgA positive cells were intense than IgG and IgM positive cells in almost all age, the findings were similar to that of Khan et al. (2008). But in the vaccinated chickens, the observation was reversed that is, the intensity of $\operatorname{IgG}$ and $\operatorname{IgM}$ positive cells were exceeds the $\operatorname{IgA}$ positive cells (Figure 6), these findings were similar to that of Jeurissen et al. (1989). In the ileum, the lining cells of the villi of all chickens were simple columnar epithelium. The villi of the ileum were long and slender; numerous goblet cells were also present among the lining epithelial cells. The scattered lymphocytes and unorganized lymphatic nodules were present in the lamina propria and core of the villi and these findings were similar that of Rahman et al. (2003). Lymphocytes in the lamina propria and intraepithelial lymphatic distribution increased in vaccinated chickens than control chickens. The effect of vaccination was also observed in the lymphatic nodule by increasing its size and increased the concentration of lymphocytic population. The findings were similar with that of many authors stated the mucosal immune system undergoes changes with ageing (Khan et al., 1996a); influenced by sex hormones (Milicevic and Milicevic, 1993; Khan et al., 1996b), and antioxidant such as selenium and vitamin E (Khan et al., 2008). In the immunohistochemical study it was found that Igs (IgA, IgG and $\operatorname{IgM}$ ) positive cells were distributed in the lamina propria, around the intestinal glands and in the core of the villi of ileum of both control and vaccinated chickens (Fig. 4a-b). The intensity of IgA positive cells were higher than the IgG and IgM positive cells in all ages of both control and vaccinated chickens (Fig. 7). This observation was similar with that of Khan et al. (2008) stated that in the ileum and cecal tonsil of broilers supplemented with selenium and vitamin E, IgA containing plasma cells were more than IgG and IgM containing plasma cells and Islam et al. (2008) reported that in the ileum of broiler and native chickens, the IgA containing plasma cells were more followed by IgM and IgG positive cells. The Igs positive cells were greatly accelerated in the vaccinated chickens than the control chickens that may be due to immunomodulatory effect of vaccine.
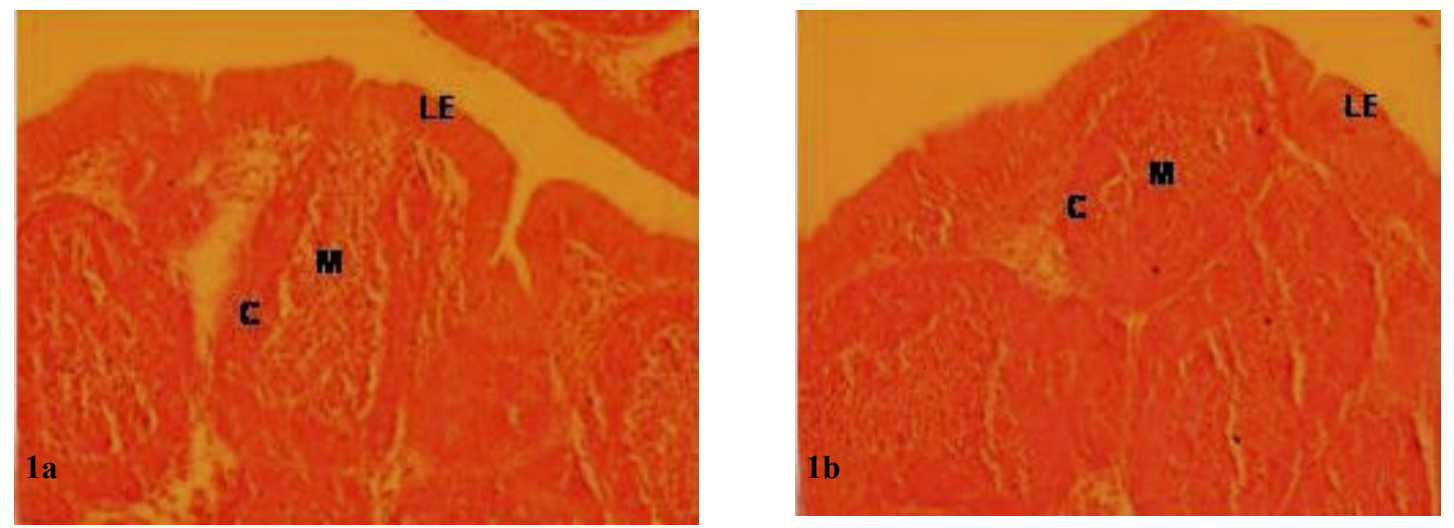

Figure 1a-b. Histological sections from the bursa of Fabricius of control (1a) and vaccinated (1b) chickens showing cortex (C), medulla (M) and lamina epithelia (LA). The cortex contained densely packed lymphocytes than the medulla. The size of follicles of the bursa and the density of lymphocytic population was increased in the vaccinated chickens (1b) than the control chickens (1a) throughout the study period. H and E stain x200. 

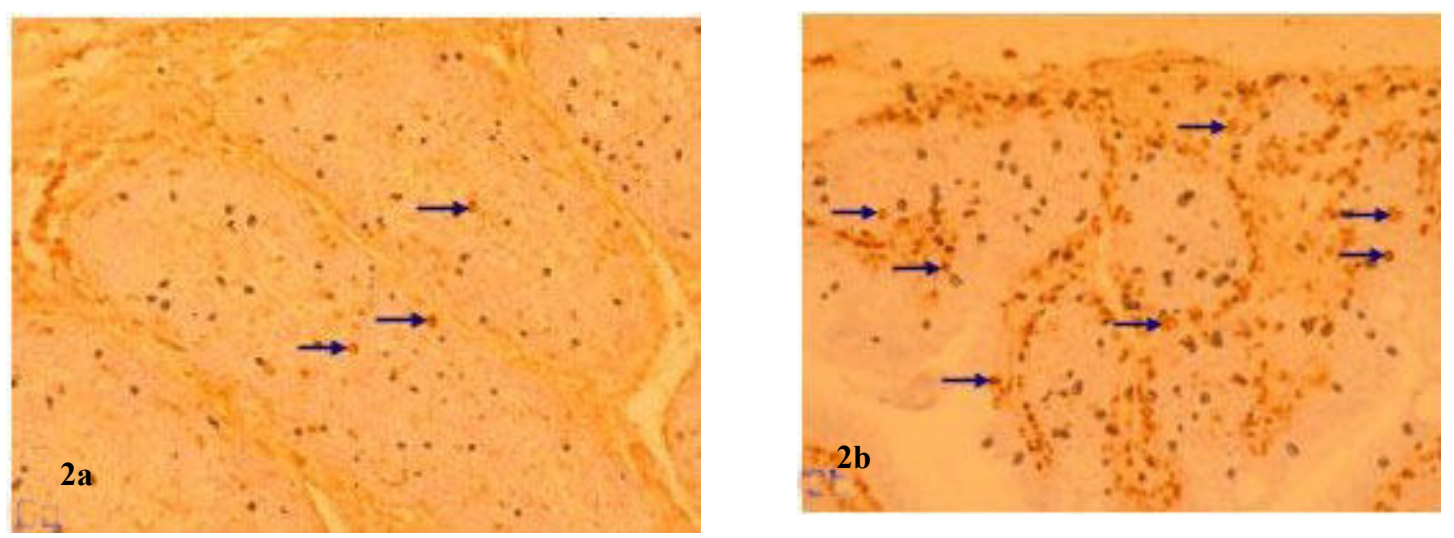

Figure 2a-b. Immunostained sections from bursa of Fabricius in the control (2a) and vaccinated (2b) chickens of 25 days old showing IgM positive cells. The IgM positive cellls were observed between the capsules and in and around the follicles, which were more in vaccinated chickens than the control chickens (arrows) x200.


Figure 3a-b. Immunostained sections from the cecal tonsils of control (3a) and vaccinated (3b) chickens at day 32 showing both IgG and IgM positive cells (arrows) which were more in vaccinated group of chickens than the control x200.
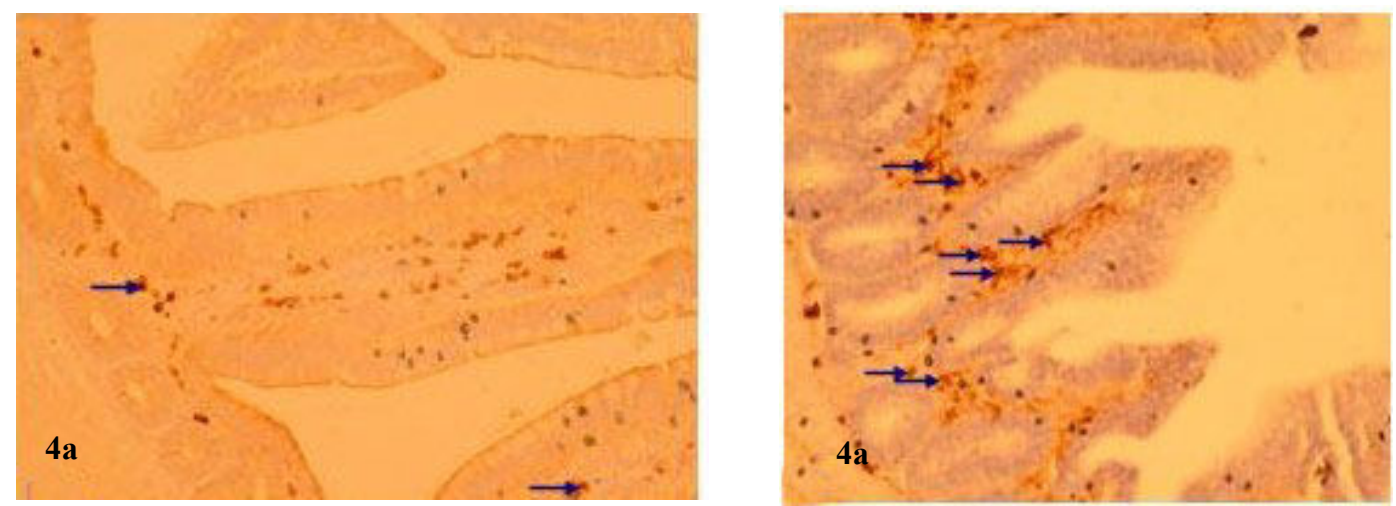

Figure 4a-b. Immunostained sections from ileum of control (4a) and vaccinated (4b) chickens of 32 days old. IgA positive cells (arrows) were more in the vaccinated chickens than the control chickens $\mathrm{x} 200$. 


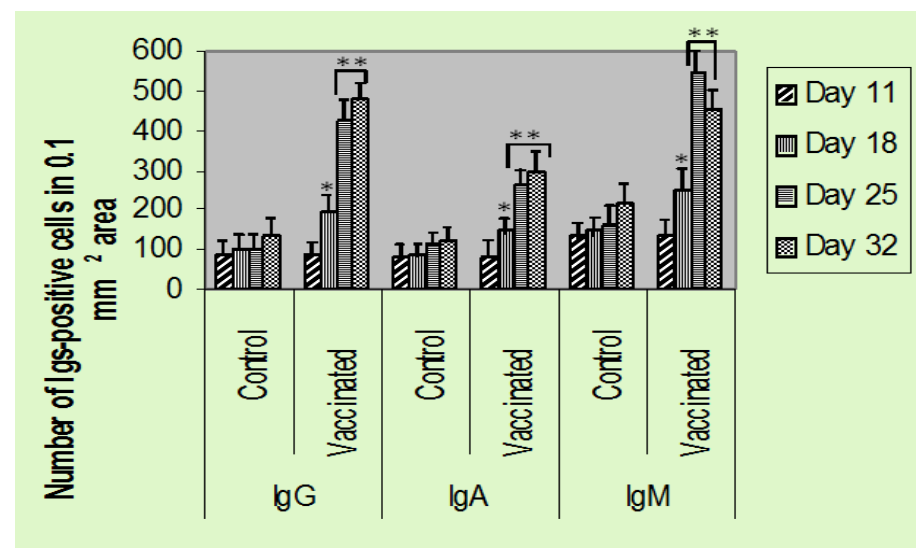

Figure 5.The frequency of Igs positive cells in the bursa of Fabricius of control and vaccinated chickens(mean $\pm \mathrm{SE}$ ) ${ }^{* *} \mathrm{p}<0.01,{ }^{*} \mathrm{p}<0.05$ vs respective control and day.The Igs positive cells (IgA, IgG,andIgM) are more in vaccinated group of chickens than the controls. IgM and IgG positive cell are more than IgA positive cells.

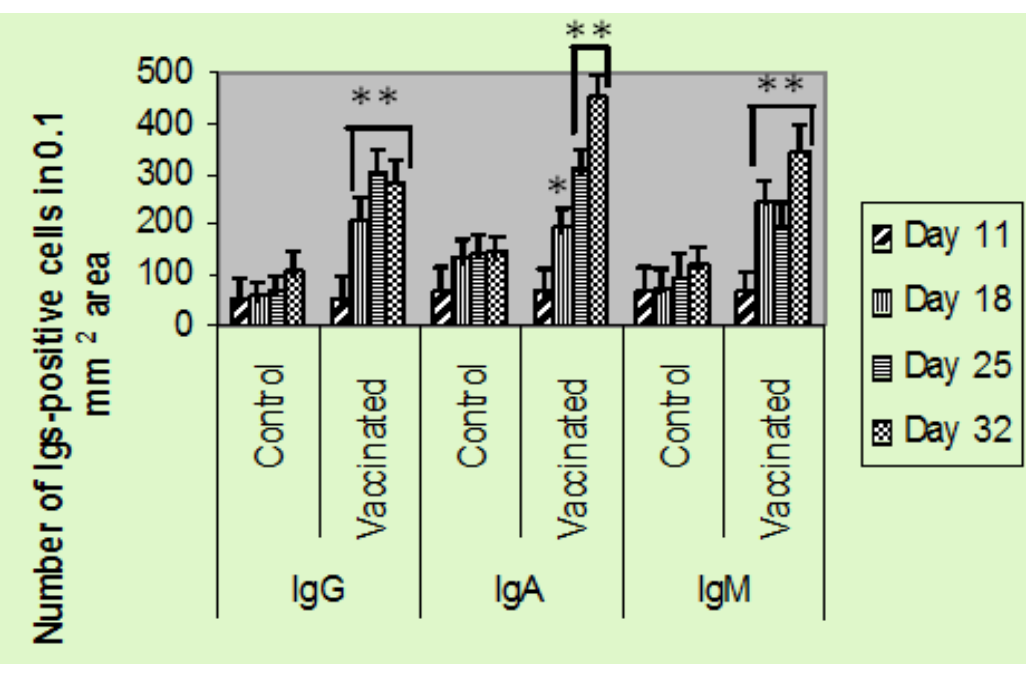

Figure 6.The frequency of Igs positive cells in the cecal tonsils of control and vaccinated chickens (mean \pm SE)

${ }^{* *} \mathrm{p}<0.01,{ }^{*} \mathrm{p}<0.05$ vs respective control and day. The Igs positive cells (IgA, IgG, and IgM) are more in vaccinated group of chickens than the controls. IgM and IgG positive cell are more than IgA positive cells. 


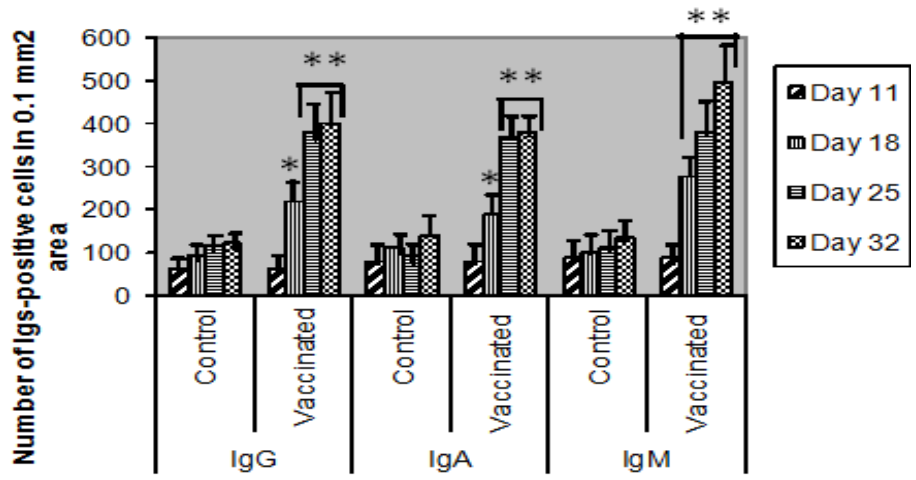

Figure 7.The frequency of Igs positive cells in the ileum of control and vaccinated chickens (mean $\pm \mathrm{SE}$ ). $*^{*} \mathrm{p}<0.01,{ }^{*} \mathrm{p}<0.05$ vs respective control and day. In all groups of chickens IgA positive cells are more in the vaccinated chickens.

\section{ACKNOWLEDGEMENT}

The research works was conducted with the financial assistance of Bangladesh Agricultural University Research System (BAURES) and Ministry of National Science, Information and Communication Technology, Bangladesh.

\section{REFERENCES}

1. Elankumaran S, Heckert RA and Mour L (2002). Pathogenesis and tissue distribution of variant strain of infectious bursal disease virus in commercial broiler chickens. Avian Diseases 46: 169-176.

2. Gridley MF (1960). Manual of histologic and special staining technique. McGraw-Hill Book Company. INC, New York, pp. 28-29, 82-83.

3. Honjo K and Hirota Y (1993). Immunohistochemical investigations of lymphocytes in the lymphoid organs of cyclophosphamide treated chickens. Journal of Veterinary Medical Science 55(5): 895-897.

4. Islam MN, Khan MZI, Jahan MR, Karim MR and Kon Y (2008). Comparative studies of mucosa and immunoglobulin (Ig) containing plasma cells in the gastrointestinal tract of broiler and native chickens of Bangladesh. Journal of Poultry Science 45: 125-131.

5. Jeurissen SHM, Janse EM, Koch G and Boer GF (1989). Postnatal development of mucosa-associated lymphoid tissues in chickens. Cell and Tissue Research 258: 119-124.

6. Khan MZI, Akter SH, Islam MN, Karim MR, Islam MR and Kon Y (2008). The effect of selenium and vitamin $\mathrm{E}$ on the lymphocytes and immunoglobulin containing plasma cells in the lymphoid organ and mucosa-associated lymphatic tissues of broiler chickens. Anatomia Histologia Embryologia 37: 52-59.

7. Khan MZI, Akter SH, Islam MN, Karim MR, Islam MR, Kon Y (2008). The effect of Selenium and Vitamin $\mathrm{E}$ on the Lymphocytes and Immunoglobulin containing-plasma cells in the lymphoid organ and MucosaAssociated lymphoid tissues of broiler chickens. AnatomiaHistologiaEmbryologia37: 52-59.

8. Khan MZI, Hashimoto Y, Iwami Y and Iwanaga T (1996b). Hormonal regulation of T-cell subsets in the oviduct: an immunohistochemical study using sex-hormone-treated chickens. Journal of Veterinary Medical Science 58: 1116-1167.

9. Khan MZI, Hashimoto Y, Iwami Y and Iwanaga T (1997). Postnatal development of B-lymphocytes and immunoglobulin-continuing plasma cells in the chicken oviduct: studies on the cellular distribution and influence of sex hormones. Veterinary Immunology and Immunopathology 56: 329-338.

10. Khan MZI, Jahan MR, Islam MN, Haque Z, Islam MR and Kon Y (2007). Immunoglobulin (Ig)-containing plasma cells in the Harderian gland in broiler and native chickens of Bangladesh. Tissue and Cell 39: 141149.

11. Khan MZL, Hashimoto Y, Konnol A, Kon Y and Iwanagal T (1996a). Development of T-lymphocyte subpopulations in the postnatal chicken oviduct. Cell and Tissue Research 284: 317-325.

12. Lukert PD and Saif Y (1991). Infectious bursal disease. In: Diseases of Poultry, (9th edn.) BW Calnek, HJ Barnes, CW Beard, WM Reid and JHW Yoder, Eds. Iowa State University Press Ames. Iowa, 648-663.

13. MilicevicZandMilicevic N (1993). Structure of the bursa of Fabricius and immune response of Brucellaabortusin the chicken after neonatal gonadectomy. Annals of Anatomy 175: 291-298. 
14. Rahman ML, Islam MR, Asaduzzaman M and Khan MZI (2003). Lymphoid tissues in the digestive tract of deshi chicken (Gallus domesticus) in Bangladesh. Pakistan Journal Biological Sciences 6(13): 1145-1150.

15. Rautenschlein S, Yehand HY and Sharma JM (2001). A comparison of the immunopathogenesis of different IBDV strains. Proceeding of the 11. International symposium on infectious bursal disease and chicken infectious anaemia, held on 16-20 July, 2000, at Rouischholzhausen, Germany, pp. 311-323.

16. Rose ME (1979). Lymphatic System, Form and Function in Birds, Vol1., Ed. by King, KS and McLelland I, Academic press, London, pp. 324-372.

17. Tanimura N, Tsumakoto K, Nakamura K, Narita M and Maeda M (1995). Association between pathogenicity of infectious bursal diseases virus and viral antigen distribution detected by immunohistochemistry. Avian Diseases 39: 9-20.

18. Vervelds L, Janse E, MargaVermeulen, Arno N andJearissen Suzan HM (1998). Induction of a local and systemic immune response using cholera toxin as vehicle to deliver antigen in the lamina propria of the chicken intestine. Veterinary Immunology and Immunopathology 62: 261-272.

19. Weibel ER (1969). Stereological principles for morphometry in electron microscopic cytology. International Review for Cytology 26: 235-302. 